\title{
Dissecting antibody-mediated protection against SARS-CoV-2
}

\section{Tomer Zohar ${ }^{1,2}$ and Galit Alter ${ }^{1 凶}$}

Understanding the properties and mechanisms by which antibodies provide protection is essential to defining immunity. Although neutralizing antibodies have been proposed as a potential key mechanism of protection against many viral pathogens, antibodies mediate additional immune functions that may have both protective and pathological consequences. Dissecting these properties against SARS-CoV-2 is likely necessary for defining metrics of immunity that will inform the design of vaccines and therapeutics and improve clinical management.

Although most individuals infected with SARS-CoV-2 experience mild-to-moderate disease, a subset fall critically ill and develop acute respiratory distress syndrome (ARDS), which is marked by neutrophil and monocyte extravasation into bronchi, cytokine storms and tissue damage from widespread inflammation. However, why some individuals succumb to infection and how humoral immunity affects the outcome remain incompletely understood.

Antibodies to SARS-CoV-2 evolve rapidly after infection and coincide with disease progression. Emerging data suggest that early SARS-CoV-2-specific antibody titres are elevated in those with severe disease ${ }^{1}$, calling into question the role of the antibody response in immunopathology. Neutralization, the ability of antibodies to directly block infection, has been implicated in protective immunity following vaccination ${ }^{2}$. However, population level studies show that most individuals who recover develop only low levels of neutralizing antibodies ${ }^{3}$, indicating that neutralizing antibodies are likely to have a relatively limited impact on resolving disease. In animal models of SARS, the passive administration of neutralizing antibodies to the virus resulted in attenuated disease $e^{4}$ and, likewise, the induction of neutralizing antibodies via immunization in animal models of COVID-19 attenuated disease ${ }^{2}$. This indicates that neutralizing antibodies may be more critical for protection against infection than for resolving disease.

Beyond neutralization, antibodies provide anti-

${ }^{\prime}$ Ragon Institute of $\mathrm{MGH}, \mathrm{MIT}$, and Harvard, Cambridge, MA, USA.

${ }^{2}$ Department of Biological Engineering, Massachusetts Institute of Technology, Cambridge, MA, USA.

凶e-mail: galter@partners.org https://doi.org/10.1038/ s41577-020-0359-5 extra-neutralizing antibody responses can enhance pathology. Thus, understanding how antibodies balance protective and potential pathogenic roles against SARS-CoV-2 is critically needed to inform therapeutic design and public health decisions.

Research focused on investigating severe disease among individuals with high antibody levels has perplexed scientists. Although sub-neutralizing antibody titres from second infections and vaccination have been linked to enhanced disease in patients with dengue, any evidence of antibody-dependent enhancement (ADE) has yet to be observed in humans infected with SARS-CoV-2. Instead, thus far, the only evidence to support a role for antibody-mediated enhancement relates to the presence of high antibody levels in individuals with severe disease ${ }^{1}$. However, antibodies may simply accrue as a biomarker of higher antigen exposure. The causal link between antibody abundance and enhancement in humans remains unclear.

Nevertheless, previous vaccine studies for SARS-CoV suggest that vaccine-induced antibodies may directly promote enhanced disease upon exposure to the virus via the activation of macrophages able to produce high levels of the chemokine CCL2 and the cytokine IL-8, resulting in lung injury ${ }^{5}$. Moreover, in vitro experiments have shown that macrophages can be infected with SARS-CoV even when they lack the ACE2 receptor that serves as the entry receptor for the virus; this occurs through antibody-mediated virus uptake via $\mathrm{Fc} \gamma \mathrm{RII}^{6}$. Although it is unlikely that such uptake results in the propagation of infection, the delivery of the virus to endosomal compartments can trigger patternrecognition receptors and induce inflammatory cascades. Conversely, in mice, the passive transfer of ADCP-inducing antibodies, rather than a neutralizing antibody alone, resulted in enhanced viral control and clearance $^{4}$. Because distinct vaccine platforms induce antibodies with different effector functions compared 


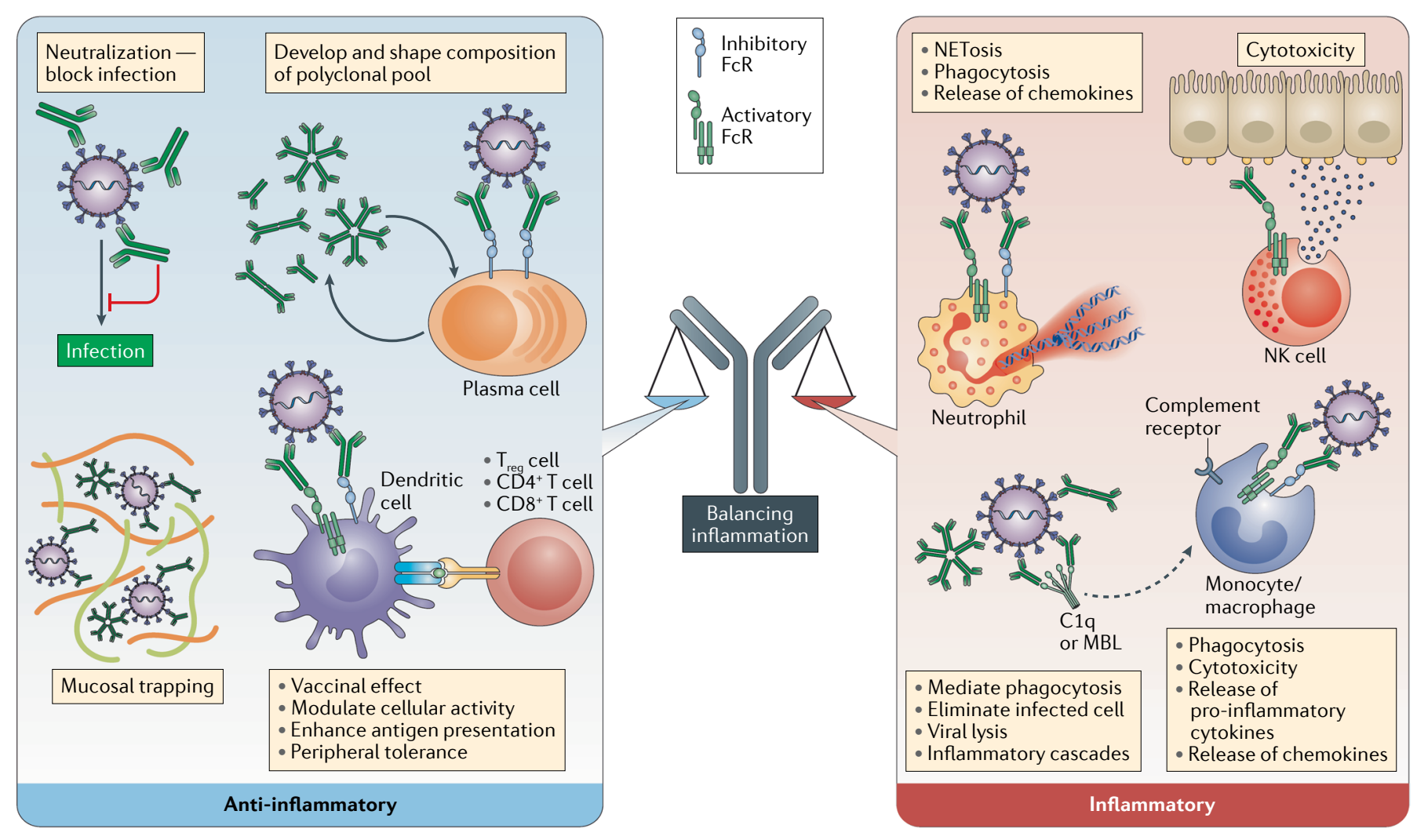

Fig. 1 Antibody functions and their contributions to inflammation. Polyclonal pools are comprised of antibodies with unique Fc structure and antigen specificity. Collectively, profiles result in different functions and downstream consequences. FcR, Fc receptor; NK, natural killer; $\mathrm{T}_{\text {reg }}$ cell, regulatory T cell.

with those induced by natural infection or those engineered into monoclonal antibodies, it is plausible that some vaccines may drive pathological and other protective antibody profiles, warranting a close analysis of vaccine-induced antibodies to ensure the deployment of a safe vaccine.

Early reports have also begun to implicate complement in disease severity in COVID-19. Over-activation of the complement cascade by the SARS-CoV-2 nucleoprotein, via the lectin pathway, resulted in enhanced lung injury in mice 7 . This study also showed that adding nucleocapsid-directed neutralizing monoclonal antibodies reduced fatality rates and lung injury. In addition to FcR expression, nearly all innate immune cells also express complement receptors, providing an additional avenue through which antibodies may activate and direct the immune system. However, whether elevated antibody titres in progressive disease drive or temper complement activation remains to be determined.

In addition to humoral immunity, emerging data point to a critical role for cellular immunity in the resolution of SARS-CoV-2 infection. Antibodies collaborate intimately with the components of cellular immunity, which is often overlooked. The recovery of two patients with agammaglobulinaemia from SARS-CoV-2 infection has led some immunologists to conclude that $\mathrm{T}$ cell immunity may be critical for resolution of infection ${ }^{8}$. However, in some cases of agammaglobulinaemia, B cells can still differentiate and result in low levels of antibodies, as in the case of one of these recovered patients. Furthermore, both patients received intravenous immunoglobin (IVIG) transfusions, potentially providing cross-reactive antibodies. Antibodies play a critical role not only in direct antiviral immunity but also in priming $\mathrm{T}$ cells via the delivery of antigens to antigen-presenting cells, in a process known as the vaccinal effect. Thus, antibodies may drive direct antiviral activity, activate adaptive immune cells via FcRs or complement receptors, and/or drive more effective priming of $\mathrm{T}$ cells.

Interestingly, in addition to being used to treat agammaglobulinaemia, IVIG is widely used as an antiinflammatory treatment for autoimmune diseases including Kawasaki syndrome. Along these lines, anecdotal transfer of convalescent plasma has been noted to have a beneficial impact on SARS-CoV-2 disease resolution'. Whether this clinical benefit is exclusively due to the supplementation of additional neutralizing or extra-neutralizing SARS-CoV-2-targeted antibodies, or because these antibodies compete for occupancy on immune complexes and drive reduced innate immune activation, remains to be determined. The antiinflammatory effects of IVIG and convalescent plasma therapy may also result in less $T$ cell exhaustion and aid the effective clearance of infected cells. It is clear that complete control and clearance likely require both blunting the infection directly and eliminating infected cells. These processes may occur collaboratively via direct antibody-mediated antiviral functions and/or their indirect role in mitigating inflammation. 
Antibodies drive a myriad of functions that both directly and indirectly interrupt infection, each with different consequences (FIG. 1). Fully appreciating both neutralizing and extra-neutralizing antibody functions in the control of SARS-CoV-2 infection will pave the way to the rational design of effective vaccines and therapeutics. Given the concern about ADE, and the remarkably broad additional potential immune-protective function of antibodies in controlling inflammation, it is critically important that vaccines will be evaluated for their pro-inflammatory and anti-inflammatory antiviral Fc functional profiles to maximize the true potential of the humoral immune system.

1. Long, Q.-X. et al. Antibody responses to SARS-CoV-2 in patients with COVID-19. Nat. Med. https://doi.org/10.1038/s41591-020 0897-1 (2020)

2. $\mathrm{Yu}, \mathrm{J}$. et al. DNA vaccine protection against SARS-CoV-2 in rhesus macaques. Science https://doi.org/10.1126/science.abc6284 (2020).

3. Wu, F. et al. Neutralizing antibody responses to SARS-CoV-2 in a COVID-19 recovered patient cohort and their implications.
Preprint at medRxiv https://doi.org/10.1101/2020.03.30.20047365 (2020).

4. Yasui, F. et al. Phagocytic cells contribute to the antibody-mediated elimination of pulmonary-infected SARS coronavirus. Virology 454-455, 157-168 (2014).

5. Liu, L. et al. Anti-spike IgG causes severe acute lung injury by skewing macrophage responses during acute SARS-CoV infection. JCI Insight 4, e 123158 (2019).

6. Jaume, M. et al. Anti-severe acute respiratory syndrome coronavirus spike antibodies trigger infection of human immune cells via a $\mathrm{pH}$ - and cysteine protease-independent Fc $\gamma \mathrm{R}$ pathway. J. Virol. 85, 10582-10597 (2011).

7. Gao, T. et al. Highly pathogenic coronavirus $\mathrm{N}$ protein aggravates lung injury by MASP-2-mediated complement over-activation. Preprint at medRxiv https://doi.org/10.1101/2020.03.29.20041962 (2020).

8. Soresina, A. et al. Two X-linked agammaglobulinemia patients develop pneumonia as COVID-19 manifestation but recover. Pediatr. Allergy Immunol. https://doi.org/10.1111/pai.13263 (2020).

9. Duan, K. et al. Effectiveness of convalescent plasma therapy in severe COVID-19 patients. Proc. Natl Acad. Sci. USA 117 9490-9496 (2020).

Author contributions

The authors contributed equally to all aspects of the article.

Competing interests

G.A. is a founder of Seromyx Inc. T.Z. declares no competing interests. 